\title{
Obstructive sleep apnoea and its therapy influence high-density lipoprotein cholesterol serum levels
}

\author{
J. Börgel*, B.M. Sanner*, A. Bittlinsky*, F. Keskin*, N.K. Bartels*, N. Buechner", \\ A. Huesing ${ }^{+}$, L.C. Rump and A. Mügge*
}

ABSTRACT: Recent studies suggest an association of obstructive sleep apnoea (OSA) with cardiovascular risk factors, such as dyslipidaemia.

The present study analyses the effects of OSA and its therapy on serum lipid concentrations in 470 OSA patients in a single centre study.

Multivariate regression showed a significant association between the apnoea-hypopnoea index and high-density lipoprotein cholesterol (HDL-C) serum levels $(n=366)$, independent of age, sex, body mass index, diabetes and lipid lowering medication. There were no independent associations with total cholesterol, triglyceride and low-density lipoprotein cholesterol serum levels. During follow-up (6 months) with effective bilevel or continuous positive airway pressure therapy in 127 patients (lipoproteins: $n=86$ ) without change in their lipid lowering therapy, the mean HDL-C serum level increased significantly by $5.8 \%$ from $46.9 \pm 15.8$ to $49.6 \pm 15.3 \mathrm{mg} \cdot \mathrm{dL}^{-1}$ (mean \pm SD).

An independent relationship was found between the change of apnoea-hypopnoea index and the change of high-density lipoprotein cholesterol or triglycerides, respectively. All patients with abnormal serum lipid/lipoprotein levels improved significantly under bilevel or continuous positive airway pressure therapy. This study demonstrates an influence of obstructive sleep apnoea and its therapy on high-density lipoprotein cholesterol levels.

KEYWORDS: Continuous positive airway pressure, hyperlipidaemia, lipid, lipoprotein, obstructive sleep apnoea

yperlipidaemia is a main risk factor for the development and progression of cardiovascular diseases. Disorders in lipid metabolism may result in early occurrence of atherosclerotic alterations [1, 2]. In addition to the well characterised pro-atherosclerotic effect of high low-density lipoprotein cholesterol (LDL-C) [3] concentrations, there is an inverse correlation between the high-density lipoprotein cholesterol (HDL-C) level and the incidence of coronary heart disease (CHD) [4]. Even a slight elevation of the HDL-C concentration can reduce the risk for CHD significantly [5].

In previous studies, an association between sleepdisordered breathing and cardiovascular diseases $[6,7]$ was detected. Besides the well described influence on blood pressure, sleep-disordered breathing may also affect other cardiovascular risk factors, for example the lipid metabolism. This latter association was recently addressed by the Sleep Heart Health Study (SHHS). In this crosssectional study in which 6,440 primary healthy subjects were screened for sleep-disordered breathing (mean respiratory disturbance index (RDI) 4.0 events $\cdot h^{-1}$ ), a significant correlation of the RDI with the total serum cholesterol levels in males and with the HDL-C and triglyceride levels in females was observed [8].

In the present study, the association between obstructive sleep apnoea and lipid serum levels was assessed in a large collective $(n=470)$ of consecutive in-patients with moderate-to-severe obstructive sleep apnoea (OSA; mean apnoeahypopnoea index $\left.(\mathrm{AHI})=28.15( \pm 22.06) \cdot \mathrm{h}^{-1}\right)$. The effect of positive airway pressure therapy on lipid-, and lipoprotein serum levels was studied in a subgroup of patients in whom the lipid lowering therapy was not changed.

\section{METHODS \\ Patients}

The patients were consecutively examined and screened for OSA between 1999 and 2001 in the sleep laboratory of the Marien-Hospital Herne, a
AFFILIATIONS

${ }^{*}$ Dept of Medicine, Cardiology and Angiology, St. Josef-Hospital/ Bergmannsheil,

"Dept of Medicine, Marien-Hospital Herne, and

+Institute for Biomedical Informatics, Ruhr-University Bochum, and \#Dept of Medicine, BethesdaHospital, Wuppertal, Germany.

\section{CORRESPONDENCE}

J. Börgel

Medical Clinic II Cardiology and Angiology

St. Josef-Hospita/Bergmannsheil Gudrunstrasse 56 44791 Bochum Germany Fax: 492345092303 E-mail: jan.boergel@ruhr-unibochum.de

Received:

November 152004

Accepted after revision:

September 132005

SUPPORT STATEMENT

This study was supported by the Ruhr-University of Bochum (FoRUM: Forschungsförderung der RuhrUniversität Bochum, Medizinische Fakultät). 
hospital of the Ruhr-University Bochum, Germany. The patients were admitted to the hospital because of a history of apnoeas or snoring or hypersomnic symptoms, such as daytime tiredness or impairment of cognitive functions. A total of 470 consecutive patients were identified with the diagnosis of OSA. The cardiovascular risk profile was determined at the time of the initial polysomnography. The assessment included a standardised questionnaire on the patient's history, physical examination, polysomnography, ECG, echocardiography, and a blood analysis (total cholesterol, triglycerides, bland-glucose and electrolytes). In 366 patients, an additional determination of serum HDL-C and LDL-C levels was available. All patients gave informed consent to participate in this study. The study protocol was approved by the local ethics committee of the Ruhr-University of Bochum.

\section{Follow-up during positive airway pressure therapy}

All patients that were treated with bilevel or continuous positive airway pressure (Bi-/CPAP) therapy due to OSA were re-evaluated by polysomnography after 6 months. A subgroup of 127 patients was extracted in whom the lipid lowering medication was kept constant during the initial visit and during the follow-up period. In 86 of these patients, HDL-C and LDL-C values were available for both visits.

\section{Polysomnography}

All patients underwent overnight polysomnography (Somnostar 4100, SensorMedics, Yorba Linda, CA, USA), according to a standardised protocol [9]. Patients were monitored with continuous polygraphic recording from surface leads for electroencephalography (C3/A1, C4/A2), bilateral electrooculography, submental and leg electromyography, electrocardiography, oxyhaemoglobin saturation (finger pulse oxymetry), chest and abdominal excursion (inductance plethysmography), nasal and oral airflow from noninvasive sensors, body position, tracheal sounds (microphone) and thoracic and abdominal respiratory movement (inductance plethysmography). Recordings were continuously supervised by a technician in order to ensure that the transducers and lead wires permitted normal positional changes during sleep. Bedtime and awakening time were at each subject's discretion. Polysomnography was determined after final waking. In the morning, patients stayed without breakfast until blood samples were taken. Polysomnography recordings were scored in $30 \mathrm{~s}$ periods for sleep, breathing and oxygenation. A breathing event during sleep was defined as abnormal if either a complete cessation of airflow lasting $\geqslant 10 \mathrm{~s}$ was seen (apnoea) or a reduction in respiratory airflow of $\geqslant 50 \%$ of the tidal volume lasting $\geqslant 10 \mathrm{~s}$ could be discerned (hypopnoea) [9]. Obstructive apnoea was defined as absence of tidal volume in the presence of paradoxical chest or abdominal wall motion. The average number of episodes of apnoea and hypopnoea per hour of sleep (AHI) was calculated. If the AHI was $\geqslant 5 \cdot h^{-1}$, associated with typical clinical features, OSA syndrome was diagnosed. Sleep was staged manually, using the methods of Rechtschaffen and Kales [10]. Patients who had pure or mainly central sleep apnoeas were excluded from the study. Further parameters such as arousal index and sleep efficiency were identified according to the recommendations of the American Sleep Disorders Association [11].

\section{Blood chemistry}

The diagnostic systems for quantitative in vitro determination of serum levels were delivered from "DiaSys" (Diagnostic Systems International $\mathrm{GmbH}$, Holzheim, Germany). Total cholesterol was determined by the "CHOD-PAP"- enzymatic photometric test ("Cholesterol FS", Diagnostic Systems International $\mathrm{GmbH}$ ). Determination of cholesterol was performed after enzymatic hydrolysis and oxidation. The colorimetric indicator was quinoneimine, which is generated from 4-aminoantipyrine and phenol by hydrogen peroxide under the catalytic action of peroxidase (Trinder's reaction) [12].

Triglyceride levels were determined by a colorimetric enzymatic test, using glycerol-3-phosphate-oxidase ("Triglyceride FS*", Diagnostic Systems International GmbH). After enzymatic splitting with lipoprotein lipase, the indicator quinoneimine (s.o.) was used as an indicator.

HDL-C was measured by an immuno-separation-based homogenous assay ("HCL-C Immuno FS"', Diagnostic Systems International $\mathrm{GmbH}$ ) and LDL-C by direct colour producing enzymatic reaction ("LDL-C Select FS"', Diagnostic Systems International $\mathrm{GmbH}$ ). These tests are homogeneous methods for HDL-C/LDL-C measurement without centrifugation steps. Antibodies against human lipoproteins are used to form antigen-antibody complexes with very low-density lipoprotein, chylomicrons and HDL-C or LDL-C in a way that only the left HDL-C or LDL-C, respectively, is selectively determined by an enzymatic cholesterol measurement [13].

\section{Statistical analysis}

Results are presented as mean $\pm \mathrm{SD}$. All reported p-values are two-tailed. The relationship between the lipid and lipoprotein serum levels and the AHI were first explored by bivariate regression analysis. To determine the independent association of the AHI on these parameters in the presence of body mass index (BMI), age, sex, diabetes and lipid lowering drugs, multiple linear regressions were calculated. Differences between untreated OSA and treated OSA after 6 months were analysed using a t-test for paired samples. Intergroup differences between AHI-quartiles were compared by ANOVA. Proportional changes of the mean values were presented as percentage. To investigate whether the change (differences between the first and second polysomnography) in lipid-/lipoprotein serum levels during the follow-up period were independent of the change in BMI a multiple regression analysis was performed. For blood analysis, the following cut-off values were defined as abnormal: total cholesterol $\geqslant 200 \mathrm{mg} \cdot \mathrm{dL}^{-1}$, triglycerides $\geqslant 180 \mathrm{mg} \cdot \mathrm{dL}^{-1}, \mathrm{HDL}$ $\mathrm{C} \leqslant 40 \mathrm{mg} \cdot \mathrm{dL}^{-1}$ and $\mathrm{LDL}-\mathrm{C} \geqslant 150 \mathrm{mg} \cdot \mathrm{dL}^{-1}$.

\section{RESULTS}

\section{Associations in untreated obstructive sleep apnoea}

Table 1 summarises the demographic findings, the cardiovascular risk profile, as well as the polysomnographic results for the full study group and for the follow-up subgroup. In this subgroup of 127 patients Bi-CPAP therapy was maintained and lipid lowering agents were not changed. Repeated determination of LDL-C and HDL-C levels was obtained in 86 patients. 
TABLE 1 Demographics of the full study cohort and the subgroups with available lipoprotein serum levels

\begin{tabular}{|c|c|c|c|c|}
\hline & \multicolumn{2}{|c|}{ Untreated OSAS } & \multicolumn{2}{|c|}{ Follow up during $\mathrm{Bi}-/$ CPAP therapy } \\
\hline & Full cohort & Subgroup (+lipoproteins) & Full cohort & Subgroup (+lipoproteins) \\
\hline Patients n & 470 & 366 & 127 & 86 \\
\hline Age yrs & $55.4 \pm 11.1$ & $55.8 \pm 11.3$ & $55.7 \pm 10.6$ & $54.9 \pm 10.4$ \\
\hline Sex female & $78(16.6)$ & $62(16.9)$ & $17(13.4)$ & $13(15.1)$ \\
\hline $\mathrm{AHI} \mathrm{h}^{-1}$ & $28.2 \pm 22.0$ & $26.8 \pm 21.7$ & $32.9 \pm 21.5$ & $30.6 \pm 20.2$ \\
\hline $\mathrm{O}_{2}$ saturation $\%$ & $92.0 \pm 4.2$ & $92.0 \pm 4.0$ & $91.4 \pm 5.4$ & $91.5 \pm 5.7$ \\
\hline Max. $\mathrm{O}_{2}$ desat. \% & $81.8 \pm 9.5$ & $82.1 \pm 9.2$ & $79.8 \pm 10.6$ & $80.2 \pm 10.2$ \\
\hline Sleep efficiency \% & $86.5 \pm 10.9$ & $86.5 \pm 10.7$ & $86.7 \pm 11.3$ & $87.3 \pm 10.5$ \\
\hline Bi-/CPAP therapy & $18 / 334(3.8 / 71.0)$ & 12/268 (3.3/73.2) & $5 / 122(3.9 / 96.1)$ & 2/84 (2.3/97.7) \\
\hline AHI (PAP ther.) $h^{-1}$ & $2.8 \pm 3.4$ & $2.8 \pm 3.5$ & $3.0 \pm 3.6$ & $2.8 \pm 2.8$ \\
\hline LDL-C s-level mg $\cdot \mathrm{dL}^{-1}$ & $148.2 \pm 36.2$ & $148.2 \pm 36.2$ & $144.5 \pm 37.8$ & $144.5 \pm 37.8$ \\
\hline \multicolumn{5}{|c|}{ Additional cardiovascular risk factors } \\
\hline Diabetes mellitus type 2 & $80(17.0)$ & $54(14.8)$ & $30(23.6)$ & $18(20.9)$ \\
\hline Arterial hypertension & $270(57.4)$ & $204(55.7)$ & $75(59.1)$ & $47(54.7)$ \\
\hline Current smoking (self rep.) & $129(27.4)$ & $108(29.5)$ & 38 (29.9) & $30(34)$ \\
\hline \multicolumn{5}{|l|}{ Cardiovascular history } \\
\hline Coronary artery disease & $55(11.7)$ & $49(13.4)$ & $16(12.6)$ & $9(10.5)$ \\
\hline Myocardial infarction & $27(5.7)$ & $26(7.1)$ & $7(5.5)$ & $4(4.7)$ \\
\hline Stroke/TIA & $22(4.7)$ & $15(4.1)$ & $8(6.3)$ & $7(8.1)$ \\
\hline Peripheral artery disease & $7(1.5)$ & $4(1.1)$ & & \\
\hline \multicolumn{5}{|l|}{ Medication } \\
\hline Insulin therapy & $10(2.1)$ & $8(2.2)$ & $2(1.6)$ & $2(2.3)$ \\
\hline
\end{tabular}

Data are presented as mean \pm SD and $n(\%)$. OSAS: obstructive sleep apnoea syndrome; Bi-/CPAP: bilevel or continuous positive airway pressure; BMI: body mass index; AHI: apnoea-hypopnoea index; AHI (PAP ther.): AHI after adjustment of positive airway pressure therapy (Bi-PAP or C-PAP); HDL-C: high-density lipoprotein cholesterol; LDL-C: low-density lipoprotein cholesterol; TIA: transient ischaemic attack; ACE-/AT1-inhibitors: angiotensin converting enzyme-inhibitor/angiotensin receptor 1inhibitor.

Bivariate correlation analysis showed a significant positive relationship between AHI and triglyceride serum levels $(\mathrm{r}=0.146, \mathrm{p}=0.002)$ and a significant negative relation between AHI and HDL-C levels $(\beta=-0.283, p<0.001)$. There was no correlation between AHI and total cholesterol or LDL-C serum levels.

ANOVA revealed a significant difference between the quartiles of AHI for HDL-C and triglycerides (fig. 1).

Adjustment for age, BMI, sex, diabetes and lipid lowering medication disclosed an independent influence of the AHI and HDL-C serum levels $(\beta=-0.195, \mathrm{p}<0.001) \quad($ table 2$)$.
Triglycerides and AHI were not related independently with a level of significance $<0.05(p=0.107)$.

\section{Follow-up during bi-level-/continuous positive airway pressure therapy}

Of 153 patients who were treated with Bi-/CPAP therapy and had a lipid determination at the time of the control polysomnography, 26 had to be excluded because their lipid lowering therapy was changed during the follow-up period. Of those, 22 received a new lipid lowering drug (mainly statins) and in four patients an existing lipid lowering medication was stopped. 

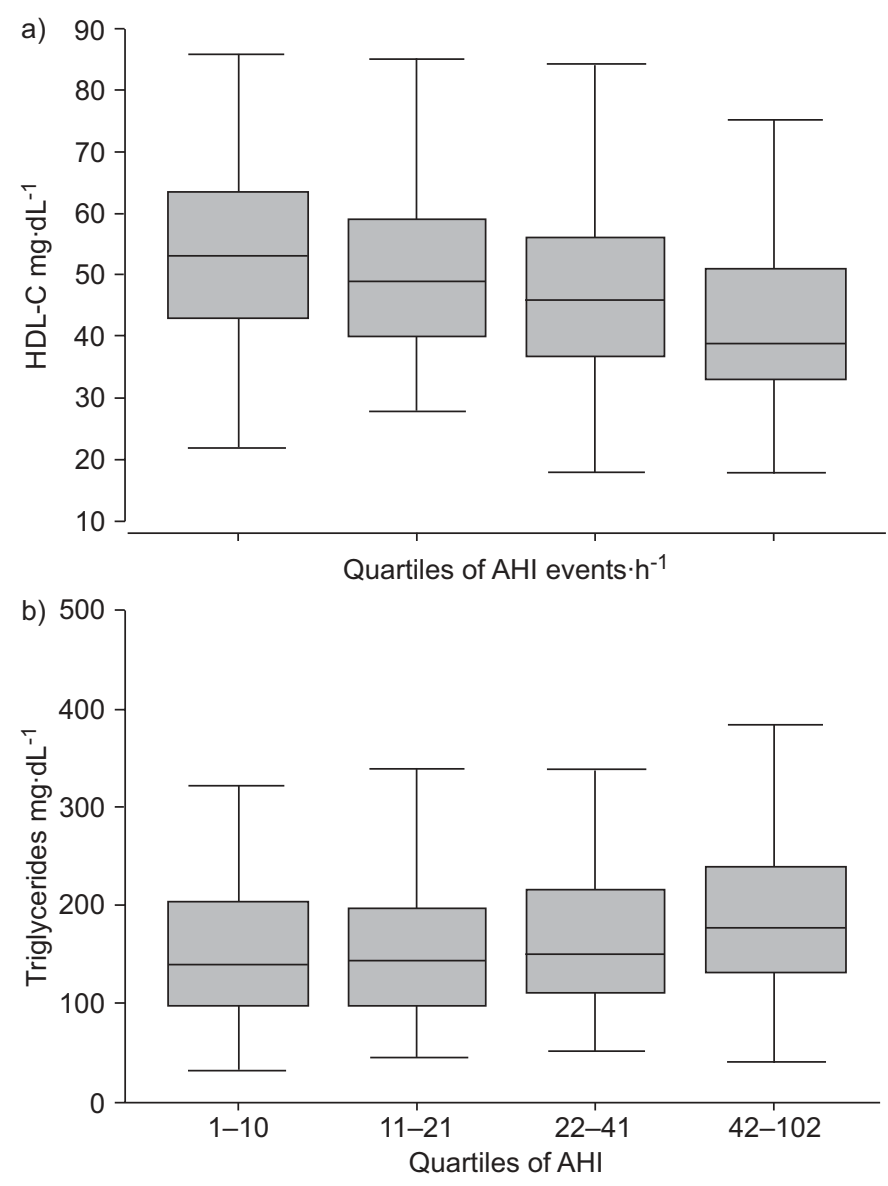

FIGURE 1. Distribution of a) high-density lipoprotein cholesterol (HDL-C; $n=366, p<0.001)$ and $b)$ triglyceride levels $(n=470, p=0.01)$ in different quartiles of the apnoea-hypopnoea index (AHI)

The demographic profile of the remaining follow-up cohort is summarised in table 1 . Only $9.4 \%(12 / 127)$ of these patients were on lipid lowering drugs at the initial enrollment (statins: $n=11$; fibrate: $n=1$ ). The doses of the lipid-lowering therapy was not changed in these patients during the 6 months of follow-up. As shown in table 1, 23.6\% of the patients $(n=30)$ had the diagnosis of diabetes mellitus, while only nine received anti-diabetic treatment. During follow-up, in six of those patients anti-diabetic treatment was changed.

The mean AHI was effectively reduced from $32.9 \pm 21.5$ to $2.2 \pm 2.3$ events $\cdot h^{-1}(p<0.001$, table 3$)$. Table 3 summarises the changes of serum lipid levels during OSA therapy. The mean HDL-C serum level increased significantly by $\sim 2.7 \mathrm{mg} \cdot \mathrm{dL}^{-1}$ $(5.8 \%, p=0.013)$. If the six patients with a change in their antidiabetic treatment during follow-up were excluded, the decrease of HDL-cholesterol remained significant $(p=0.015)$. The mean serum concentrations of total cholesterol $\left(-3.4 \mathrm{mg} \cdot \mathrm{dL}^{-1} ;-1.44 \%\right)$, triglycerides $\left(-8.6 \mathrm{mg} \cdot \mathrm{dL}^{-1} ;-4.9 \%\right)$ and LDL-C $\left(-5.5 \mathrm{mg} \cdot \mathrm{dL}^{-1} ;-3.8 \%\right)$ decreased, but these changes did not reach statistical significance. On follow-up, the mean BMI remained unchanged $(-0.9 \%, \mathrm{p}=\mathrm{NS})$.

Multivariate regression analysis revealed a significant relationship between the improvement of $\mathrm{AHI}(\Delta \mathrm{AHI})$ and changes of HDL-C ( $\Delta$ HDL-C) and triglyceride ( $\Delta$ triglycerides) levels.

\begin{tabular}{llc} 
TABLE 2 & $\begin{array}{l}\text { Multivariate regression between apnoea- } \\
\text { hypopnoea index (AHI) and total cholesterol, } \\
\text { triglyceride-, HDL-C and LDL-C serum levels }\end{array}$ \\
\cline { 2 - 3 } & \multicolumn{2}{c}{ AHI adjusted } \\
\hline & 0.005 & p-value \\
\hline & 0.076 & 0.927 \\
Total cholesterol & -0.195 & 0.107 \\
Triglycerides & 0.045 & 0.001 \\
HDL-C & 0.434 \\
LDL-C & \\
\hline &
\end{tabular}

These results remained significant after adjustment for $\Delta \mathrm{BMI}$ (table 4).

In the follow-up group, 91 had a cholesterol level $\geqslant 200 \mathrm{mg} \cdot \mathrm{dL}^{-1}\left(\mathrm{n}=67 \geqslant 220 \mathrm{mg} \cdot \mathrm{dL}^{-1}\right), 45 \mathrm{had}$ triglyceride levels $\geqslant 180 \mathrm{mg} \cdot \mathrm{dL}^{-1}, 41$ had HDL-C levels $\leqslant 40$ and 38 patients had LDL-C levels $\geqslant 150 \mathrm{mg} \cdot \mathrm{dL}^{-1}$. As shown in figure 2, all the lipids/lipoproteins improved significantly in patients with initial pathological levels.

\section{DISCUSSION}

This study demonstrates the following four points. 1) The number of nocturnal hypopnoeas or apnoeas in patients with obstructive sleep-disordered breathing is associated independently with low HDL-C levels. 2) Bi-/CPAP therapy significantly increases the mean HDL-C serum levels by $\sim 5.8 \%$ within 6 months of treatment. 3) There is an independent $(\triangle \mathrm{BMI})$ and significant relation between the changes of AHI and HDL-C or triglycerides, respectively, indicating some reversibility of the initial dyslipidaemia under Bi-/CPAP therapy. 4) All mean lipid/lipoprotein serum levels improve significantly under Bi-/CPAP therapy in OSA patients with initial abnormal lipid/lipoprotein serum level values.

The current results are partially confirmatory to previous work. In the SHHS, an independent correlation was observed between the respiratory disturbance index and HDL-C levels in females only. In males, a minor, but significant association was seen only for the total cholesterol levels. Interestingly, these correlations were more evident in the age group $<65$ yrs as compared with elderly patients [8]. The current study shows important differences to the SHHS. 1) The mean age in the current study is younger (55.4 versus $62.1 \mathrm{yrs}$ ). 2) The present study enrolled in-patients with moderate-to-severe OSA (mean AHI 28 events $\cdot h^{-1}$ ) in contrast to the SHHS, which screened initially healthy patients without or with mild-to-moderate sleep-disordered breathing (mean RDI $=4$ events $\cdot h^{-1}$ ). 3) The cardiovascular risk profile is on average worse in the cohort of the current study.

Although there are significant differences in HDL-C levels between males and females $\left(46.5 \mathrm{mg} \cdot \mathrm{dL}^{-1}\right.$ versus $55.8 \mathrm{mg} \cdot \mathrm{dL}^{-1}$, 
TABLE 3 Effect of Bi-/CPAP therapy on polysomnographic data, lipids and body mass index (BMI) in patients with obstructive sleep apnoea

Untreated OSA After 6 months of $\mathrm{Bi}-/$ CPAP $\quad$ Mean difference $(95 \% \mathrm{Cl}) \quad$ p-value

therapy

Polysomnography
AHI events $\cdot \mathrm{h}^{-1}$
Mean $\mathrm{O}_{2}$ saturation
Maximum $\mathrm{O}_{2}$ desaturation
Arousal index
Sleep efficiency
Lipids
Total cholesterol
Triglycerides
HDL-C
LDL-C
BMI

$\begin{array}{cc}32.9 \pm 21.5 & 2.2 \pm 2.3 \\ 91.4 \pm 5.4 & 93.9 \pm 1.9 \\ 79.8 \pm 10.6 & 88.0 \pm 7.1 \\ 19.1 \pm 8.0 & 7.9 \pm 6.1 \\ 86.7 \pm 11.3 & 86.3 \pm 13.6 \\ & \\ 223.5 \pm 41.4 & 220.30 \pm 39.4 \\ 175.1 \pm 102.3 & 166.2 \pm 87.77 \\ 46.9 \pm 15.8 & 49.6 \pm 15.25 \\ 144.5 \pm 37.8 & 138.99 \pm 37.1 \\ 31.6 \pm 5.9 & 31.4 \pm 5.4\end{array}$

$\begin{array}{rr}-30.7(-26.8--34.6) & <0.001 \\ 2.8(3.5-1.5) & <0.001 \\ 8.1(10.5-5.8) & <0.001 \\ -11.2(-6.1--16.3) & <0.001 \\ -0.4(2.9--3.9) & 0.784 \\ & \\ -3.4(3.6--10.0) & 0.351 \\ -8.6(6.2--23.5) & 0.116 \\ 2.7(4.9-0.6) & 0.013 \\ -5.5(2.0--13.0) & 0.151 \\ -0.9(0.15--0.6) & 0.229\end{array}$

Data are presented as mean \pm SD unless otherwise stated. Bi-/CPAP: bilevel or continuous positive airway pressure; Cl: confidence interval; AHI: apnoea-hypopnoea index; HDL-C: high-density lipoprotein cholesterol; LDL-C: low-density lipoprotein cholesterol.

$\mathrm{p}<0.001$ ) in the current study group, the influence of OSA on HDL-C levels is independent of sex as shown in table 2. In contrast to the SHHS, the use of lipid lowering medication and the diagnosis diabetes, both important confounders, are considered in the statistical analysis of the present study.

A new finding is that Bi-/CPAP therapy has a potentially beneficial effect on lipid serum levels. In the follow-up cohort a significant increase in the HDL-cholesterol serum concentration of $\sim 5.8 \%$ was observed within 6 months of therapy. The change in AHI and the change in HDL-C serum levels correlated with high significance, demonstrating the reversibility of, and also confirming the association between, lipid serum levels and AHI in untreated OSA.

A previous study [14] reports that CPAP therapy reduced the LDL-C serum levels in 69 OSA patients. In contrast to the current study, only patients with elevated LDL-C plasma levels $>130 \mathrm{mg} \cdot \mathrm{dL}^{-1}$ before CPAP therapy were included.

\begin{tabular}{|c|c|c|c|c|}
\hline \multirow[t]{3}{*}{ TABLE 4} & \multicolumn{4}{|c|}{$\begin{array}{l}\text { Multivariate regression between therapy-induced } \\
\text { changes of apnoea-hypopnoea index }(\Delta \mathrm{AHI}) \\
\text { and lipids }\end{array}$} \\
\hline & & $\Delta \mathbf{A H I}{ }^{\#}$ & \multicolumn{2}{|c|}{ Adjusted for $\Delta \mathrm{BMI}$} \\
\hline & $\beta$ & p-value & $\boldsymbol{\beta}$ & $p$-value \\
\hline$\Delta$ Cholesterol & 0.096 & 0.316 & 0.052 & 0.590 \\
\hline$\Delta$ Triglycerides & 0.372 & 0.019 & 0.317 & 0.044 \\
\hline$\Delta H D L-C$ & -0.112 & 0.001 & -0.112 & 0.001 \\
\hline$\Delta$ LDL-C & 0.153 & 0.159 & 0.136 & 0.212 \\
\hline
\end{tabular}

\# : initial value while obstructive sleep apnoea was untreated minus value after 6 months of bilevel or continuous positive airway pressure therapy; BMI: body mass index; HDL-C: high-density lipoprotein cholesterol; LDL-C: low-density lipoprotein cholesterol.
Therefore, these findings do not contradict the present authors' results, since in the subgroup of patients with initial abnormal LDL-C levels $>150 \mathrm{mg} \cdot \mathrm{dL}^{-1}$ a significant reduction under $\mathrm{Bi}-/$ CPAP therapy was also found (fig. 2). However, these improvements of lipid levels may be amplified by a regressionto-the-mean effect.

However, in another meta-analysis of two randomised placebo-controlled trials of short-term CPAP-treatment (1 month) in OSA patients a significant fall of total cholesterol of $-0.28 \mathrm{mmol} \cdot \mathrm{L}^{-1}(-4.9 \%)$ in 107 OSA patients was observed, while in a control group of 106 patients with sub-therapeutic CPAP-therapy changes were not significant. Nonfasting triglyceride levels were not affected in both groups. HDL-C and LDL-C levels were not determined [15].

The link between severity of OSA and lipid metabolism remains to be defined. It may be speculated that this link is related to a confounding factor, obesity, which has a strong association with both OSA and lipid disorders. Moreover, low HDL-C levels, high triglyceride levels and obesity are associated with each other as part of the metabolic syndrome [16]. However, the SHHS as well as the present study demonstrated a correlation between $\mathrm{AHI}$ and lipid plasma levels that was independent of the BMI. This observation suggests that factors other than obesity alone may be involved.

This/these factor(s) may be related to an increased sympathetic nerve system activity, as it has been consistently reported for patients with OSA [17-19]. A direct link between the adrenergic system and lipid levels was shown before: previous pharmacological studies using $\alpha$ - and $\beta$-adrenoceptor blockers disclosed an influence of the sympathetic nervous system on the HDL-C and triglyceride serum levels: prazosin increases HDL-C levels and reduces triglyceride levels [20, 21], while propanolol [19] and metoprolol administration [21] are associated with a significant increase in serum levels of triglycerides and with a decrease in the HDL-C serum 

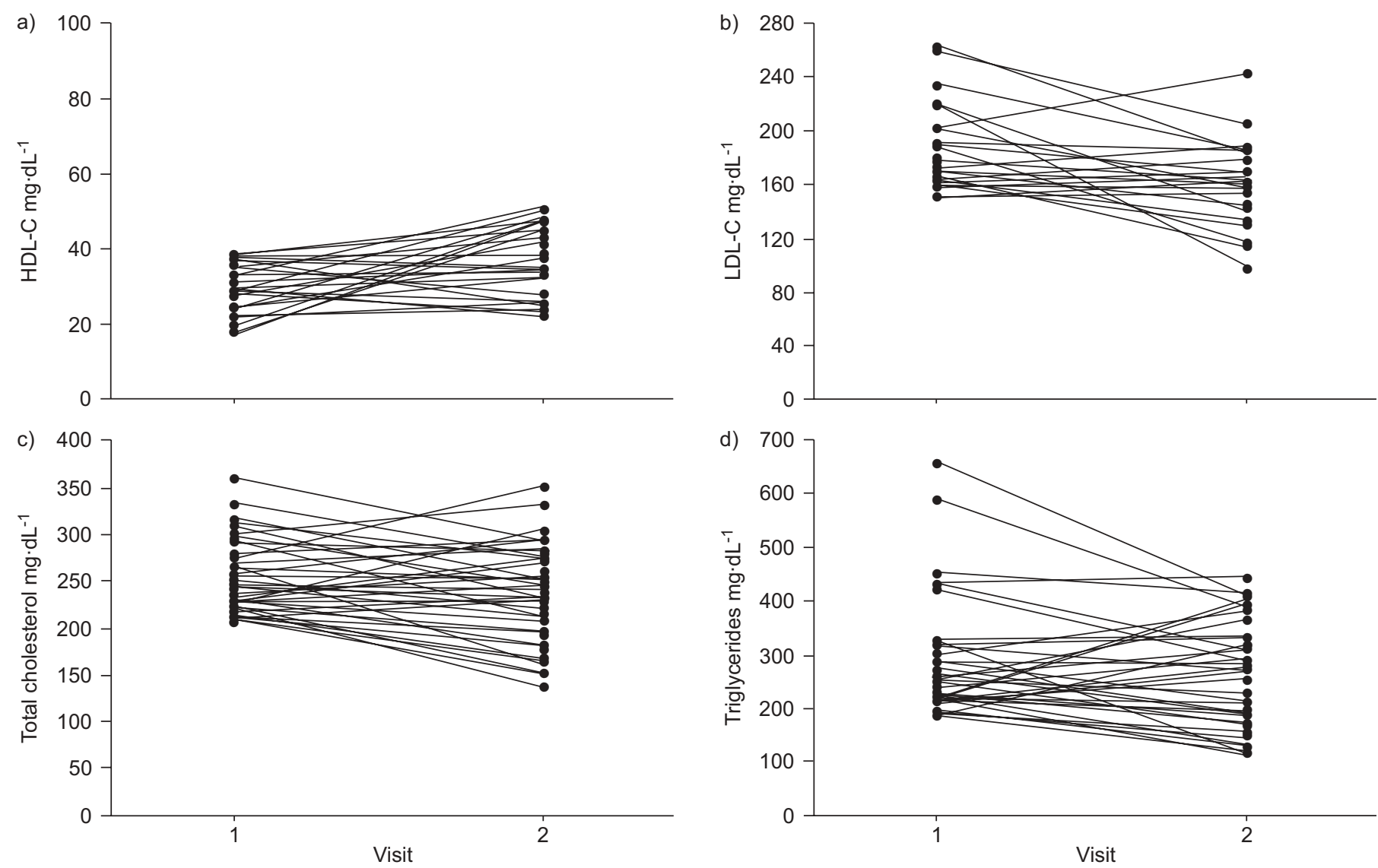

FIGURE 2. Changes of lipid serum levels of a) high-density lipoprotein cholesterol (HDL-C), b) low-density lipoprotein cholesterol (LDL-C), c) total cholesterol and d) triglycerides under bi-level-/continuous positive airway pressure therapy in patients with initial abnormal lipid profile. The development of the mean (abnormal) serum level and the t-test (comparison between first and second visit) was: a) HDL-C, 31.6-36.8 $\mathrm{mg} \cdot \mathrm{mL}^{-1}(\mathrm{p}<0.001)$; b) LDL-C, 188.8-160.4 $\mathrm{mg} \cdot \mathrm{mL}^{-1}$ ( $p<0.001$ ); c) total cholesterol, 242.3$228.9 \mathrm{mg} \cdot \mathrm{mL}^{-1}(\mathrm{p}=0.001)$; and d) triglycerides, $300.7-252.3 \mathrm{mg} \cdot \mathrm{mL}^{-1}(\mathrm{p}=0.01)$.

concentration. In accordance with these results, terbutaline, a $\beta_{2}$-receptor agonist, raised the HDL-C level in healthy subjects [23]. Furthermore, adrenalin [24] and cortisol [25], which are elevated in OSA patients [17], modify the expression of the lipoprotein lipase, which has a key role in the synthesis of HDL-C [26]. To summarise, these findings suggest that any status with a chronic elevated sympathetic activity may lower HDL-C and increase triglyceride serum levels.

The pathomechanism lipid metabolism modification by Bi-/ CPAP therapy is unknown. On the one hand, the reduction of sympathetic nerve activity [27] may account for an improved lipid profile. On the other hand effective treatment of OSA may result in increased physical activity and a reduction of hypersomnolence during the day [28]. It is well known that physical activity can also improve lipid serum levels. A metaanalysis of 52 exercise training trials of $>12$ weeks duration including 4,700 subjects demonstrated an average increase in HDL-C levels of $4.6 \%$ and reduction of triglycerides of $3.7 \%$ $[29,30]$. Although the patients from the present study did not receive a special training programme, the increase of the mean HDL-C level was $5.8 \%$.

As mentioned in the introduction, even a mild improvement of lipid levels can reduce the risk for CHD significantly [5]. It has not yet been demonstrated that consequent therapy of OSA reduces the incidence of cardiovascular disease, but the current results indicate that this therapy reduces the risk profile.

\section{Limitations}

A disadvantage of the current study is certainly the missing information regarding physical activity in the patients. Therefore, an important confounding factor is not present in the multivariate analysis. Furthermore, waist/hip ratio would have improved the information about the fat distribution, which is an individual confounder for dyslipidaemia within the determination of the BMI. These aspects will need to be considered in future studies. Another limitation is that the study did not establish a control group, treated with e.g. subtherapeutic CPAP. However, there is a controversy about control groups who are treated with subtherapeutic CPAPtherapy, since even subtherapeutic CPAP therapy has a substantial effect on the AHI and sleep structure and therefore is no placebo treatment.

\section{Conclusion}

The current study demonstrates that the severity of OSA associated independently with low HDL-C serum levels. The present authors could further demonstrate a significant positive effect of $\mathrm{Bi}-/ \mathrm{CPAP}$ therapy on the mean HDL-C 
serum level and on all lipid/lipoprotein concentrations in OSA patients with initial abnormal serum level values. The fact that the present study could show a highly significant relationship between the change in AHI and the change in HDL-C serum levels during $\mathrm{Bi}-/ \mathrm{CPAP}$ therapy demonstrates a partial reversibility of, and thus confirms, the consequences of untreated OSA.

These observations may help to explain the increased rate of cardiovascular disease in obstructive sleep apnoea patients and underline the importance of bi-level-/continuous positive airway pressure treatment.

\section{ACKNOWLEDGEMENTS}

The authors would like to thank M. Haske and M. BurmannUrbaneck for support in patient recruitment.

\section{REFERENCES}

1 Gordon T, Castelli WP, Huortland MC, Kannel WB, Dawber TR. High density lipoprotein as a protective factor against coronary heart disease. The Framingham Study. Am J Med 1977; 62: 707-714.

2 Schulte H, Assmann G. Results of the "Münster Prospective Cardiovascular" Study. Soz Praventivmed 1988; 33: 32-36.

3 Chang MY, Lees AM, Lees RS. Low-density lipoprotein modification and arterial wall accumulation in a rabbit model of atherosclerosis. Biochemistry 1993; 32: 8518-8524.

4 Gordon DJ, Rifkind BM. High-density lipoprotein - the clinical implications of recent studies. N Engl J Med 1989; 321: 1311-1316.

5 Assmann G, Schulte H, von Eckardstein A, Huang Y. High-density lipoprotein cholesterol as a predictor of coronary heart disease risk. The PROCAM experience and pathophysiological implications for reverse cholesterol transport. Atherosclerosis 1996; 124: 11-20.

6 Shahar E, Whitney CW, Redline S, et al. Sleep-disordered breathing and cardiovascular disease: cross-sectional results of the Sleep Heart Health Study. Am J Respir Crit Care Med 2001; 163: 19-25.

7 Lavie P, Herer P, Peled R, et al. Mortality in sleep apnea patients: a multivariate analysis of risk factors. Sleep 1995; 18: $149-57$.

8 Newman AB, Nieto FJ, Guidry U, et al. Relation of sleepdisordered breathing to cardiovascular disease risk factors: the Sleep Heart Health Study. Am J Epidemiol 2001; 154: 50-59.

9 Sleep-related breathing disorders in adults: recommendations for syndrome definition and measurement techniques in clinical research. The Report of an American Academy of Sleep Medicine Task Force. Sleep 1999; 22: 667-689.

10 Rechtschaffen A, Kales A. A manual of standardized terminology techniques and scoring systems for sleep stages of human subjects. Washington, DC, USGovernment Printing Office, 1968.

11 EEG arousals. scoring rules and examples: a preliminary report from the Sleep Disorders Atlas Task Force of the American Sleep Disorders Association. Sleep 1992; 15: 173-184.

12 Deeg R, Ziegenhorn J. Kinetic enzymatic method for automated determination of total cholesterol in serum. Clin Chem 1983; 29: 1798-1802.

13 Nauck M, Maerz W, Wieland M. Immunoseparation-based homogenous assay for HDL-cholesterol compared with three homogenous and two heterogenous methods for HDL cholesterol. Clin Chem 1998; 44: 1443-1451.

14 Buechner J, Zidek W, Esser M, Haske M, Sanner BM. Obstructive sleep apnea syndrome. Effects of therapy on dyslipidemia. Somnologie 2001; 5: 97-103.

15 Robinson GV, Pepperell JCT, Segal HC, Davies RJO, Stradling JR. Circulating cardiovascular risk factors in obstructive sleep apnoea: data from randomised controlled trials. Thorax 2004; 59: 777-782.

16 Castelli WP, Cooper GR, Doyle JT, et al. Distribution of triglyceride and total, LDL and HDL cholesterol in several populations: a cooperative lipoprotein phenotyping study. J Chronic Dis 1977; 30: 147-169.

17 Narkiewicz K, Somers VK. Sympathetic nerve activity in obstructive sleep apnoea. Acta Physiol Scand 2003; 177: 385-390.

18 Flechter EC, Mille J, Schaaf JW, Fletcher JG. Urinary catecholamines before and after tracheostomy in patients with obstructive sleep apnea and hypertension. Sleep 1987; 10: 35-44.

19 Marrone O, Riccobono L, Salvaggio A. Catecholamines and blood pressure in obstructive sleep apnea syndrome. Chest 1993; 103: 722-727.

20 Leren P, Foss PO, Helgeland A, Hjermann I, Holme I, Lund-Larsen PG. Effect of propranolol and prazosin on blood lipids. The Oslo Study. Lancet 1980; 2: 2-4.

21 Lowenstein J. Effects of prazosin on serum lipids in patients with essential hypertension: a review of the findings presented at the Satellite Symposium on coronary heart disease, hypertension and other risk factors, Milan. Am J Cardiol 1984; 53: 21A-23A.

22 Ferrara LA, Marotta T, Rubba P, De Simone B, Leccia G, Soro S. Effects of alpha-adrenergic and beta-adrenergic receptor blockade on lipid metabolism. Am J Med 1986; 80: 104-108.

23 Hooper PL, Woo W, Visconti L, Pathak DR. Terbutaline raises high-density-lipoprotein-cholesterol levels. $N$ Engl J Med 1981; 305: 1455-1457.

24 Marotta T, Ferrara LA, Di Marino L, et al. Factors affecting lipoprotein lipase in hypertensive patients. Metabolism 1995; 44: 712-718.

25 Ottosson M, Vikman-Adolfsson K, Enerback S, Olvercrona G, Bjorntorp P. The effects of cortisol on the regulation of lipoprotein lipase activity in human adipose tissue. J Clin Endocrinol Metab 1994; 79: 820-825.

26 Eisenberg S. High density lipoprotein metabolism. J Lipid Res 1984; 25: 1017-1058.

27 Hedner J, Darpo B, Ejnell H, Carlson J, Caidahl K. Reduction in sympathetic activity after long-term CPAP treatment in sleep apnoea: cardiovascular implications. Eur Respir J 1995; 8: 222-229.

28 Jenkinson C, Davies RJ, Mullins R, Stradling JR. Comparison of therapeutic and subtherapeutic nasal continuous positive airway pressure for obstructive sleep apnoea: a randomised prospective parallel trial. Lancet 1999; 353: 2100-2105.

29 Leon A, Sanchez O. Response of blood lipids to exercise training alone or combined with dietary intervention. Med Sci Sports Exerc 2001; 33: Suppl. 6, S502-S515.

30 Leon A, Sanchez O. Meta-analysis of the effects of aerobic exercise training on blood lipids. Circulation 2001; 104 $414-415$. 